\title{
Chronic Epidural Hematoma: Still a Rare Entity?
}

\author{
Manpreet Singh Banga ${ }^{1}$ Sandeep B.V. ${ }^{1} \quad$ Sourabh Dixit ${ }^{2}$ \\ ${ }^{1}$ Department of Neurosurgery, Vydehi Institute of Medical Sciences \\ and Research Centre, Bangalore, Karnataka, India \\ ${ }^{2}$ Department of Neurosurgery, Bhopal Memorial Hospital and \\ Research Center, Bhopal, Madhya Pradesh, India \\ Address for correspondence Manpreet Singh Banga, MCh, \\ Department of Neurosurgery, Vydehi Hospital, \#82, EPIP Area, \\ Whitefield, Bangalore 560066, Karnataka, India \\ (e-mail: dr.msbanga@gmail.com).
}

\begin{abstract}
Keywords

- epidural hematoma

- uncal herniation

- neurodeficits

- head injury

- craniotomy

Introduction Extradural hematoma diagnosed more than 14 days after head injury is classified as a chronic extradural hematoma (CEDH). In the present study, we presented a series of 8 patients with CEDH in a span of 6 months.

Materials and Methods In this article, we reported 8 cases of CEDH who presented to Nil Ratan Sircar Medical College, Kolkata, West Bengal, India, within a span of 6 months.

Discussion Extratemporal epidural hematomas (EDHs) are often due to venous bleeding from the diploic veins or dural sinuses or to delayed rupture of a middle meningeal pseudoaneurysm. In these cases, cerebrospinal fluid may redistribute from the lateral ventricles and thus allowing room for the enlarging hematoma producing vague neurological symptoms and signs. The incidence rate of CEDH reported in the literature ranges from 3.9 to $30 \%$ of all EDHs. Computed tomography (CT) scan in CEDH often shows a low-density center surrounded by a high-density margin. Calcification of the displaced dura mater may also occur. Some are identified incidentally, whereas others are diagnosed when investigating for persistent and/or progressive neurological symptoms. Symptomatic CEDH should be surgically evacuated and has an excellent outcome the earlier it is done. In patients with no or mild symptoms, normal neurological status, and a small-sized CEDH spontaneous resolution may be expected.

Conclusion In the post-CT era, it is always said that CEDH is a rare entity. However, in developing countries we still encounter a large number of such cases and the question arises whether $\mathrm{CEDH}$ is still a rare entity.
\end{abstract}

\section{Introduction}

Acute epidural hematomas (EDHs) usually become evident clinically within hours after head injury by producing progressive neurological deterioration. The classical features are an initial period of loss of consciousness (LOC) after injury and a lucid interval followed by a rapid progressive period of unconsciousness, with motor and sensory failures ending fatally within several hours if surgical evacuation is not achieved. ${ }^{1}$ If the hematoma is located outside the temporal fossa the diagnosis

published online April 15, 2021
DOI https://doi.org/ $10.1055 / \mathrm{s}-0041-1727555$ ISSN 0973-0508 may be delayed and the expected sequence of rapid uncal herniation may not be seen. Extradural hematoma diagnosed more than 14 days after head injury is classified as a chronic extradural hematoma (CEDH). ${ }^{2}$ In the post-computed tomography (CT) era it is always said that CEDH is a rare entity. However, in developing countries like India we still encounter a large number of such cases and the question arises whether CEDH is still a rare entity? We present a series of 8 patients with CEDH who presented to Nil Ratan Sircar Medical College and Hospital, Kolkata, West Bengal, India, within a span of 6 months. (c) 2021. Neurotrauma Society of India.

This is an open access article published by Thieme under the terms of the Creative Commons Attribution-NonDerivative-NonCommercial-License, permitting copying and reproduction so long as the original work is given appropriate credit. Contents may not be used for commercial purposes, or adapted, remixed, transformed or built upon. (https://creativecommons.org/licenses/by-nc-nd/4.0/). Thieme Medical and Scientific Publishers Pvt. Ltd. A-12, 2nd Floor, Sector 2, Noida-201301 UP, India 


\section{Case Reports}

\section{Case 1}

A 28-year-old female presented to our hospital with history of fall from moving vehicle 20 days back. She had two episodes of vomiting after the incidence with no LOC. The patient had persistent holocranial headache which was partially relieved with medications. Her general physical examination revealed healing abrasions over the left frontal region. The patient was neurologically intact. A CT scan was performed which showed left frontal EDH which was surgically evacuated ( $\mathbf{- F i g . 1}$ ). Postoperative period was uneventful and patient was discharged with no neurological deficits.

\section{Case 2}

A 19-year-old boy was involved in an automobile accident 18 days back with history of transient LOC for approximately 30 seconds. He was taken to a local doctor who treated him conservatively. Due to persistent headache he presented to our hospital. He was neurologically intact with no signs of raised intracranial pressure. A CT scan was performed which showed left parietal EDH with hypodense center and hyperdense margins (-Fig. 2). He was advised surgery but the patient was not willing for the same.

\section{Case 3}

A mentally retarded male patient aged 30 years presented to our hospital with history of progressive weakness of all four limbs since past 6 months. Patient had history of multiple falls due to his mental illness. At presentation, the patient was conscious, power all four limbs $3 / 5$, and plantar extensor bilaterally. His CT scan showed EDH in the right frontoparietal,

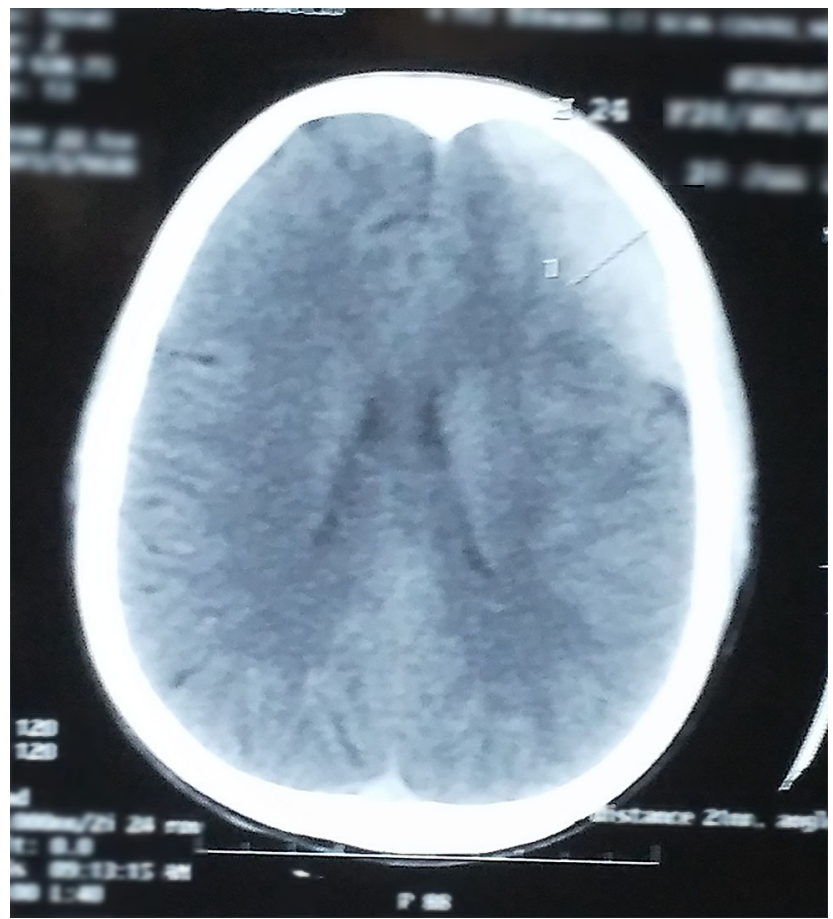

Fig. 1 Computed tomography (CT) brain of the patient with left frontal epidural hematoma (EDH). left frontal, and left parietal region with calcified walls with severe mass effect ( -Fig. 3). Patient underwent evacuation of EDH with removal of calcified membranes. Intraoperatively, the dura was densely calcified and could be partially removed. Patient was discharged with minimal improvement but with no added neurodeficits.

\section{Case 4}

An 11-year-old male child was brought to our hospital with history of fall from height 1 month back. He had two

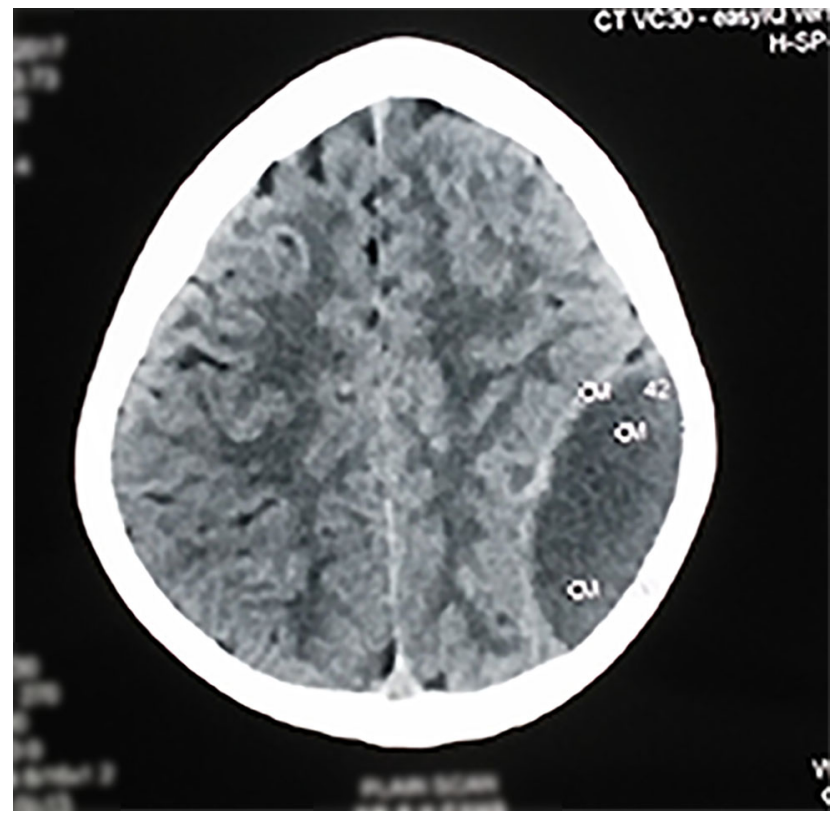

Fig. 2 Computed tomography (CT) brain of the patient with left parietal epidural hematoma (EDH) with hypodense center and hyperdense margins.

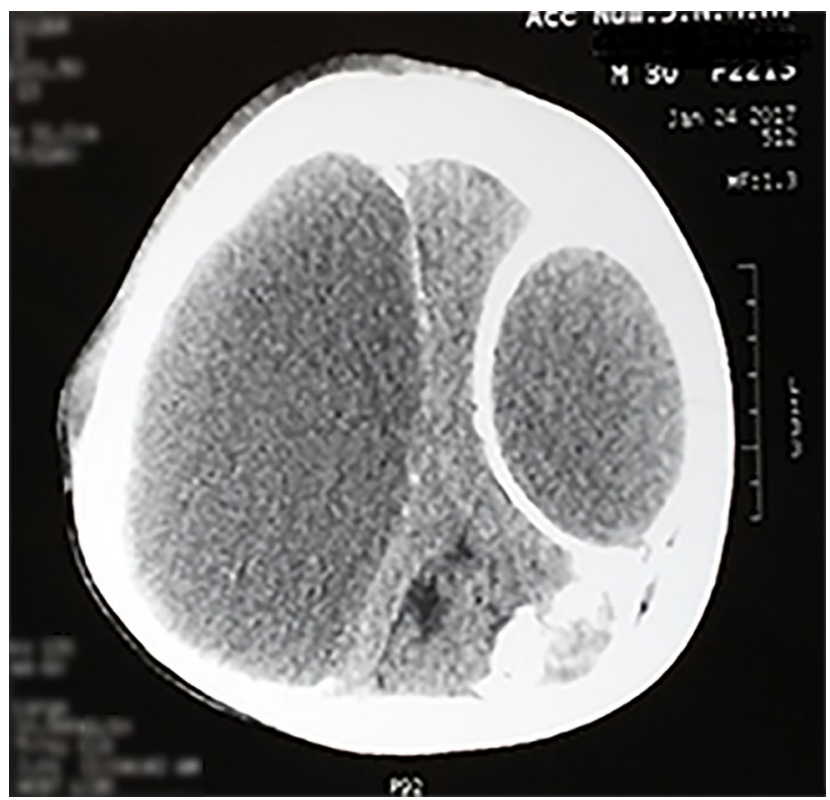

Fig. 3 Computed tomography (CT) brain of the patient showing epidural hematoma $(E D H)$ in the right frontoparietal, left frontal, and left parietal region with calcified walls with severe mass effect. 
episodes of vomiting following this episode. Patient was taken to a local doctor where he was treated conservatively. The parents brought the child to our hospital with complaints of depression in the left posterior part of the skull. On examination the child was conscious and oriented with no gross neurologic deficits. Local examination showed mild depression on left high parietal region. CT scan brain was done which showed left parietal EDH with depressed fracture ( - Fig. 4). Patient underwent elevation of depressed fracture and evacuation of EDH. Patient was discharged in satisfactory condition.

\section{Case 5}

An 8-year-old male child with history of fall from swings while playing 20 days back presented to our outpatient department. He had a brief period of LOC and one episode of vomiting at the time of injury. Following the injury, patient showed to a local doctor and was prescribed some medications. However, the child complained of persistent headache despite taking medications. On examination child was conscious and oriented with no gross neurological deficit. CT brain was done in our hospital which showed right parietal EDH which was resolving (-Fig. 5). Patient was operated in view of large size and mass effect accounting to chronic headache. He was discharged after surgery in satisfactory condition with no added neurodeficits.

\section{Case 6}

A 32-year-old female patient with alleged history of assault 28 days back presented to our hospital with complaints of giddiness since the time of injury. Her husband reported that she has occasional irrelevant behavior with forgetfulness

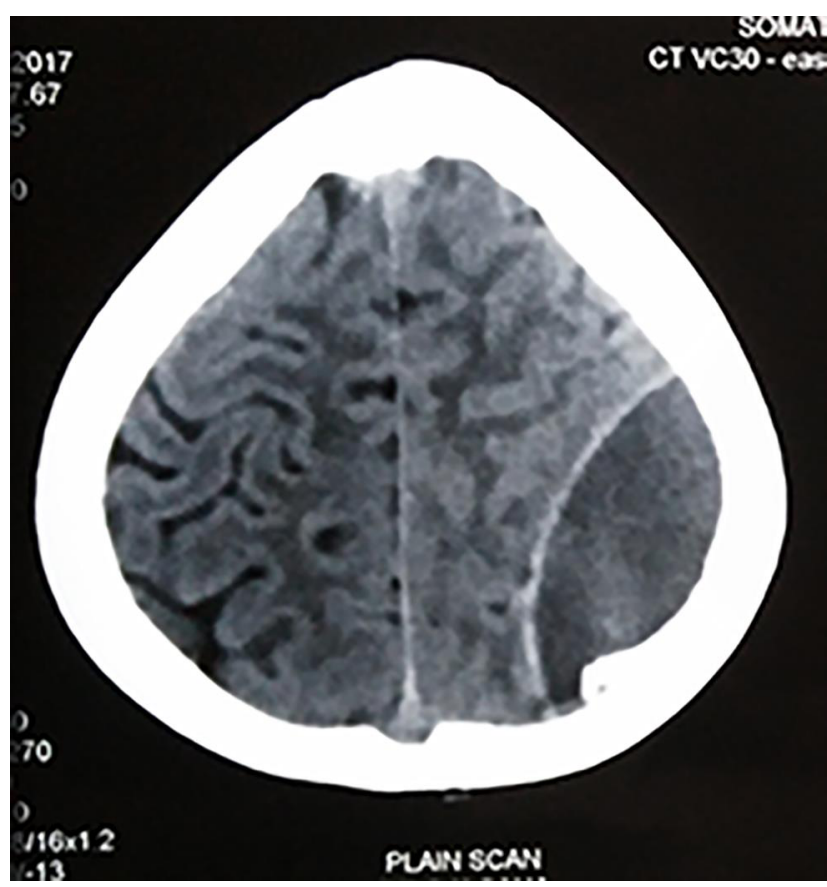

Fig. 4 Computed tomography $(\mathrm{CT})$ brain of the patient showing left parietal epidural hematoma (EDH) with depressed fracture. for 15 days. On examination the patient had a healed laceration over the right frontal region with no neurodeficits. Her mini-mental status examination was also under normal limits. CT brain was done which showed a right frontal EDH with local mass effect ( - Fig. 6). Right frontal craniotomy with evacuation of CEDH was performed. Postoperative period was uneventful and patient was discharged with no added neurodeficits.

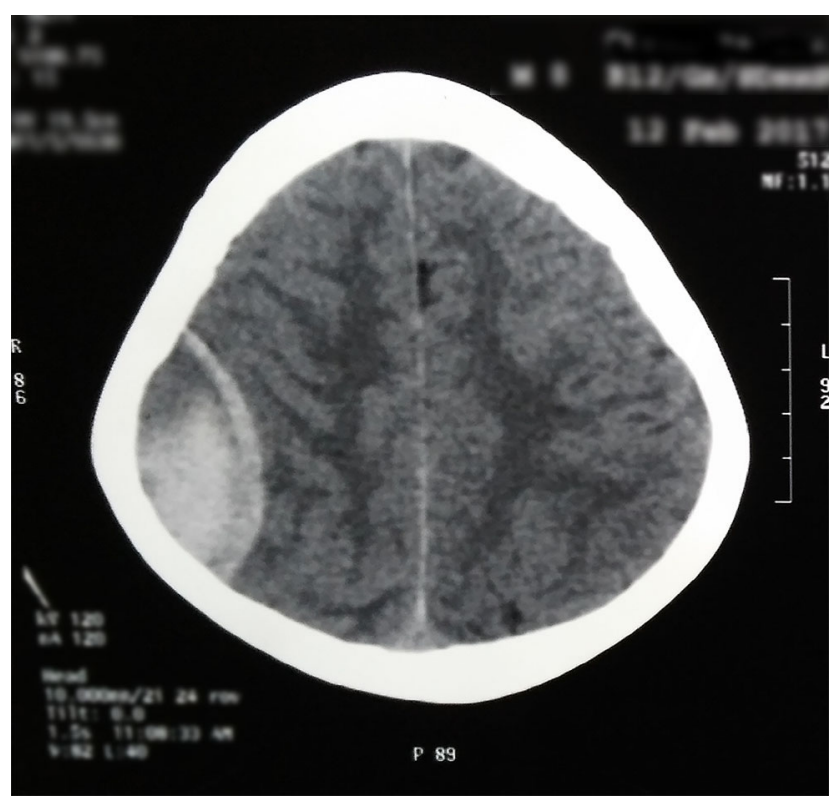

Fig. 5 Computed tomography (CT) brain of the patient showing right parietal epidural hematoma $(E D H)$ with mixed density hematoma and hyperdense rim.

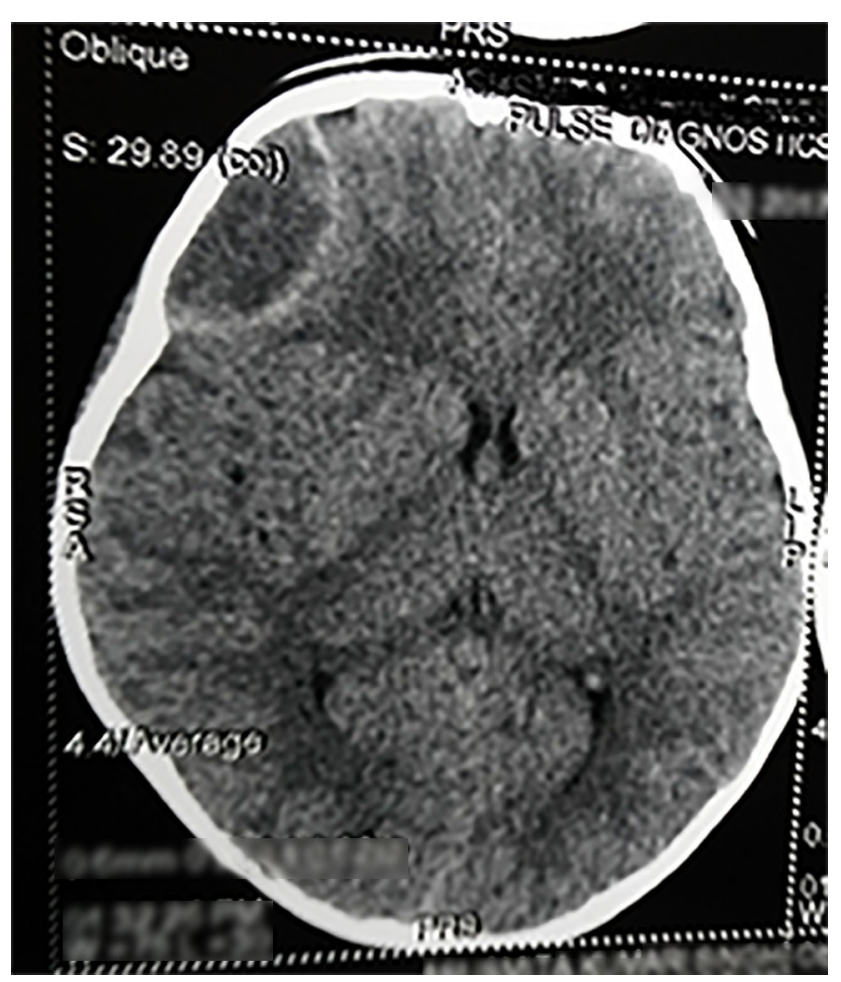

Fig. 6 Computed tomography (CT) brain of the patient showing right frontal epidural hematoma (EDH) with hypodense hematoma. 


\section{Case 7}

A 14-year-old male child with history of fall from tree 19 days back presented to our hospital with history of two episodes of abnormal jerky movements of all four limbs followed by brief period of altered sensorium in the past 15 days. Patient had no history of LOC after fall. Patient also complained of mild holocranial headache since the time of injury. On examination the child was conscious and oriented with no gross neurological deficit. He had a right below elbow cast for a fracture after the fall. CT brain was done which showed left parietal EDH with mixed density of hematoma within it ( - Fig. 7). Patient was advised surgery but the parents were not willing for the surgery.

\section{Case 8}

A 28-year-old male patient was brought to our emergency department with history of injury to head due to unknown mechanism 15 days back. The patient was found unknown on road side and was taken to a local hospital where no neurosurgical facility was available. Once he was recognized his relative brought him to our hospital. On examination, patient had bilateral resolving black eye with multiple healing facial lacerations. He was in altered sensorium with strongly localizing to pain. CT brain was done which showed bilateral frontal EDH with mixed density hematoma within it with right frontal contusion with diffuse edema and midline shift to right (-Fig. 8). He was operated and evacuation of bilateral EDH was done. Intraoperatively, patient had no bleed from the midline sinus. Patient had a long stay in the hospital and was discharged from the hospital in satisfactory condition with persistent neurodeficits.

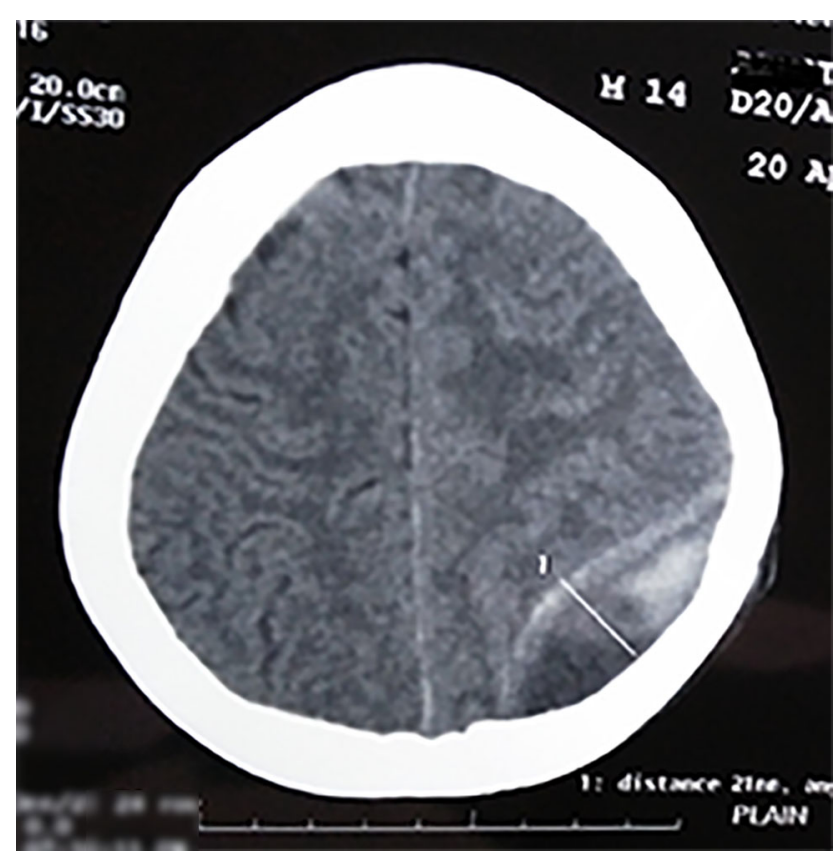

Fig. 7 Computed tomography (CT) brain of the patient showing left parietal epidural hematoma (EDH) with mixed density of hematoma.

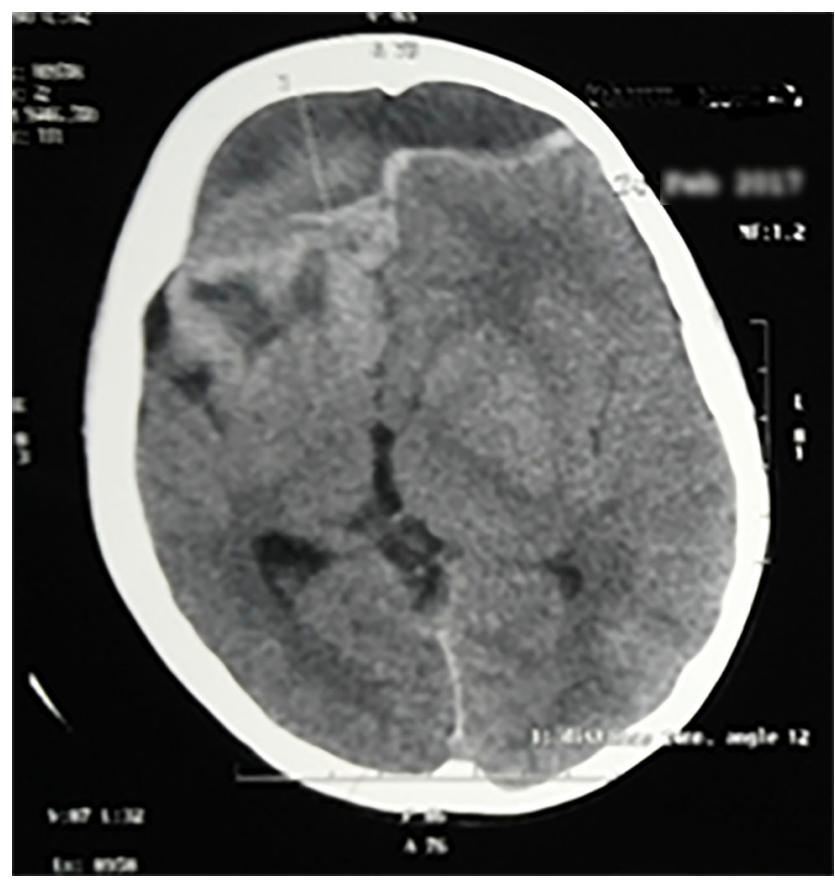

Fig. 8 Computed tomography $(C T)$ brain of the patient showing bilateral frontal epidural hematoma $(E D H)$ with mixed density hematoma within it with right frontal contusion with diffuse edema and midline shift to right.

\section{Discussion}

EDHs are usually located in the temporal fossa $(\sim 57-83 \%$ of cases) followed by frontal fossa ( $11 \%$ of cases), in the occipital region ( $7 \%$ of cases), at the vertex (2-8\% of cases), and in the posterior fossa (4-10\% of cases).,4 When located outside the temporal fossa, the usual rapidly progressive neurological deterioration caused by transtentorial uncal herniation does not occur and hence an EDH may not be suspected clinically.

Temporal epidural bleeding, usually originates from the middle meningeal artery causing rapid blood collection and early medial uncal displacement. This leads to progressive obtundation whether or not a lucid interval has been present and a high mortality rate is common. ${ }^{5}$ In contrast, extratemporal EDHs are often due to venous bleeding from the diploic veins or dural sinuses ${ }^{3,6}$ or to delayed rupture of a middle meningeal pseudoaneurysm. ${ }^{7}$ In these extratemporal cases cerebrospinal fluid may redistribute from the lateral ventricles and thus allowing room for the enlarging hematoma. Thus, the neurological symptoms and signs are usually vague and often late. ${ }^{8,9}$ Children particularly tend to demonstrate slow neurological deterioration, perhaps because their dura is loosely adhered to the inner table of skull and gets easily stripped off and distributes the pressure of the hematoma over a larger area. ${ }^{10}$

Unlike subdural hematoma, which if identified more than 21 days after injury is termed as chronic subdural hematoma, there is no consensus on the precise time-based definition of CEDH. ${ }^{11,12}$ There are various definition of $\mathrm{CEDH}$ in 
the literature. Sparacio et $\mathrm{al}^{13}$ used the term CEDH for those extradural hematomas operated $>48$ hours after injury and Clavel et $\mathrm{al}^{14}$ defined CEDH as EDH which are diagnosed $>72$ hours after injury. Bullock and van Dellen ${ }^{15}$ used the term CEDH as the EDH with injury to surgery interval of at least 7 days. Iwakuma and Brunngraber, ${ }^{16}$ however, adopted anatomopathological criterion defining CEDH as those EDH operated more than 13 days after injury and was similar to that of Zuccarello et al. ${ }^{17}$ Recently, Bradley ${ }^{2}$ has defined CEDH based on hemoglobin breakdown products on magnetic resonance imaging as extradural hematomas identified more than 14 days after head injury. This definition seems to be more scientific, recent, and evidence-based.

The incidence rate of CEDH reported in the literature ranges from $3.9^{17}$ to $30 \%{ }^{16}$ of all EDHs. It occurs more commonly in the younger ( $<40$ years) age groups. ${ }^{11}$ In our series, all 8 patients were below the age of 40 years. The pathogenetic mechanisms that can be invoked to explain chronicity in extra-axial hematomas include the presence of associated skull fractures, frontally located hematomas, age-related diffuse cerebral atrophy, venous source of bleeding, and traumatic arteriovenous fistulae of meningeal vessels. ${ }^{18} \mathrm{CT}$ scan in CEDH often shows a low-density center surrounded by a high-density margin. ${ }^{19,20}$ The mechanism of this rim enhancement is explained by granulation tissue forming a fibrovascular neomembrane on the outer surface of the dura mater in addition to the natural enhancement of the displaced dura itself. ${ }^{19}$ Calcification of the displaced dura mater may also occur. ${ }^{20}$ In our series, six patients had hyperdense rim with low-density center and one patient had calcified margins of the dura intraoperatively.

Some of the CEDHs are identified incidentally, whereas others are diagnosed when investigating for persistent and/or progressive symptoms like headache, dizziness, nausea, vomiting, memory impairment, weakness of limbs, and disturbance of consciousness. Symptomatic CEDH should be surgically evacuated and has an excellent outcome the earlier it is done. However, in patients with no or mild symptoms, normal neurological status, and a small-sized CEDH without any mass effect spontaneous resolution may be expected. A watchful wait may be appropriate in such cases, but implies a high cost of serial scans and long hospitalization. ${ }^{12}$ Surgical evacuation should be considered if CEDH is observed not to be naturally absorbed during serial scans even if the patient's condition is good, because of the likelihood of calcification. ${ }^{21}$ All our patients had persistent and/or progressive symptoms/signs during presentation. We operated on seven out of the eight patients as one patient was not willing for surgery. With the advancement in surgical techniques, neuroanesthesia, and intensive care units, it does not seem to be judicious to wait for large acute hematomas to become chronic in case of hematomas due to sinus bleed.

The mortality and morbidity rates in CEDH cases reported in other series are known to be very low except a single patient of Zhang et al. ${ }^{22}$ In our series, one patient with calcification of CEDH wall had minimal improvement after surgery probably due to inadequate brain expansion. The patient with diffuse brain injury with bifrontal CEDH also had delayed and partial improvement due to the diffuse nature of brain injury along with the CEDH.

\section{Conclusion}

Outcome of surgical management of symptomatic CEDHs is usually excellent, though smaller ones can be managed conservatively. A watchful wait may be appropriate in small CEDH cases, but implies a high cost of serial scans and long hospitalization. Any patient with head injury, even if alert, is having mild persistent symptoms and/or signs should undergo a CT scan to rule out CEDH. In the current era of advanced neuroimaging, it is always said that CEDH is a rare entity but the question arises whether in developing country where there is a shortage of specialists and facilities, especially in rural areas, is this really true.

\section{Funding}

None.

\section{Conflict of Interest}

None declared.

\section{References}

1 Grant WT. Chronic extradural hematoma. Report of a case of hematoma in anterior cranial fossa. Bull Los Angeles Neurol Soc 1944;9:156-162

2 Bradley WG Jr. Hemorrhage and hemorrhagic infections in the brain. Neuroimaging Clin N Am 1994;4(4):707-732

3 Borzone M, Gentile S, Perria C, Rivano C, Rosa M. Vertex epidural hematomas. Surg Neurol 1979;11(4):277-284

4 Hooper RS. Extradural haemorrhages of the posterior fossa. Br J Surg 1954;42(171):19-26

5 McLaurin RL, Ford LE. Extradural hematoma: statistical survey of forty -seven cases. J Neurosurg 1964;21:364-371

6 Gruskiewicz J, Doron Y, Peyser E. Frontal extradural hematomas. Surg Neurol 1976;5(2):122-128

7 Garza-Mercado R, Campa H. Extradural hematoma due to traumatic pseudoaneurysm of middle meningeal artery. Surg Neurol 1978;9(2):103-105

8 Fager CA. Subacute epidural hematoma. Surg Clin North Am 1958;38(3):877-883

9 Trowbridge WV, Porter RW, French JD. Chronic extradural hematomas. AMA Arch Surg 1954;69(6):824-830

10 Hawkes CD, Ogle WS. Atypical features of epidural hematoma in infants, children, and adolescents. J Neurosurg 1962;19: 970-980

11 Liu Y, Wang Y, Song T, et al. Chronic epidural haematoma: a report of 10 cases and review of the literature. J Clin Neurosci 1999;6(5):412-415

12 de Oliveira Sillero R, Zanini MA, Gabarra RC. Large chronic epidural hematoma with calcification: a case report. J Trauma 2008;64(6):1619-1621, discussion 1621

13 Sparacio RR, Khatib R, Chiu J, Cook AW. Chronic epidural hematoma. J Trauma 1972;12(5):435-439

14 Clavel M, Onzain I, Gutierrez F. Chronic epidural haematomas. Acta Neurochir (Wien) 1982;66(1-2):71-81

15 Bullock R, van Dellen JR. Chronic extradural hematoma. Surg Neurol 1982;18(4):300-302

16 Iwakuma T, Brunngraber CV. Chronic extradural hematomas. A study of 21 cases. J Neurosurg 1973;38(4):488-493 
17 Zuccarello M, Fiore DL, Pardatscher K, Trincia G, Andrioli GC. Chronic extradural haematomas. Acta Neurochir (Wien) 1983;67(1-2):57-66

18 Quartey GR, Gabriel YH, Tchang S. Chronic extradural hematoma presenting with subgaleal mass. Can J Neurol Sci 1980;7(1):71-72

19 Handa J, Handa H, Nakano Y. Rim enhancement in computed tomography with chronic epidural hematoma. Surg Neurol 1979;11(3):217-220
20 Lanksch W, Grumme Th, Kazner E, Computed Tomography in Head Injuries. New York: Springer-Verlag;1979:16-22

21 Chang JH, Choi JY, Chang JW, Park YG, Kim TS, Chung SS, Chronic epidural hematoma with rapid ossification. Childs Nerv Syst 2002;18(12):712-716

22 Zhang W, Zhang W, Gao Z, Wang J. Gigantic ossified chronic epidural haematoma and contralateral postoperative subdural haematoma: a case report and literature review. $\mathrm{Br} \mathrm{J}$ Neurosurg 2015;29(1):85-86 